\title{
Cosmic-ray positrons from mature gamma-ray pulsars
}

\author{
L. Zhang ${ }^{1,2}$ and K. S. Cheng ${ }^{2}$ \\ 1 Department of Physics, Yunnan University, Kunming, China \\ 2 Department of Physics, University of Hong Kong, Hong Kong, China
}

Received 12 May 2000 / Accepted 19 December 2000

\begin{abstract}
We consider a possible contribution of mature $\gamma$-ray pulsars (with ages of $\geq 10^{5}$ yrs) to cosmic ray positrons. Within the framework of the $\gamma$-ray pulsar outer gap model, $\mathrm{e}^{ \pm}$pairs in the pulsar magnetosphere are produced by the cascade of $\mathrm{e}^{ \pm}$pairs through synchrotron radiation of the return current from the outer gap. A good fraction of these cascade $\mathrm{e}^{ \pm}$pairs are reflected by the hard X-rays from the polar cap via resonant scattering and escape from the pulsar through the light cylinder. The escaped pairs are accelerated to relativistic energies in the pulsar wind driven by low-frequency electromagnetic waves. Using Monte Carlo simulations, we generate a sample of the mature $\gamma$-ray pulsars in our Galaxy and calculate the positron production rate from these pulsars. In a simple "leaky box" model, we calculate the ratio of cosmic-ray positrons to total electrons. Our result indicates that the pulsar contribution to the cosmic ray positrons peaks at about $60 \mathrm{GeV}$ and the observed $\mathrm{e}^{+} /\left(\mathrm{e}^{-}+\mathrm{e}^{+}\right)$ ratio can be explained in this model.
\end{abstract}

Key words. stars: pulsars: general - acceleration of particles - ISM: cosmic rays

\section{Introduction}

Although observed cosmic-ray positrons have been considered to be secondary products of nuclear interactions of cosmic ray with the interstellar medium, the conventional models of cosmic-ray propagation (leaky box and diffusion models) cannot explain the observed positron fraction unless one assumes a harder interstellar nucleon spectrum (see, for example, Berezinsky et al. 1990; Mori 1997). Before the 1990s, the observed cosmic-ray positron fraction between $0.05 \mathrm{GeV}$ and $50 \mathrm{GeV}$ shows that (i) the observed positron fraction below $10 \mathrm{GeV}$ is essentially explained by the secondary origin in the simple leaky-box model and (ii) there is a significant excess of positrons at energies around $10 \mathrm{GeV}$ and above, compared to the fraction expected from the secondary sources. However, the observed data on the cosmic positrons were essentially contaminated by proton events producing large positron fluxes. Since then, the experimental technique has been essentially improved. Recent observation with CAPRICE94 (Boezio et al. 2000) shows that the observed positron spectrum and the positron fraction below about $10 \mathrm{GeV}$ are consistent with a pure secondary origin in the diffusion model of cosmic ray propagation. However, the positron data at energies between 1 and $50 \mathrm{GeV}$, measured with the HEAT balloon-borne instrument (Coutu et al. 1999), suggest that a small additional positron component may

Send offprint requests to: L. Zhang, e-mail: lzhang@bohr.physics.hku.hk be present that cannot be explained by a purely secondary production mechanism. It should be pointed out that there is still some room for an additional positron component because of limiting statistics at high energies. We plotted the observed cosmic-ray positron fraction in Fig. 3. Theoretically, the fraction of secondary positrons was calculated using different propagation models of cosmic rays (e.g. Protheroe 1982). A new calculation of the cosmic-ray secondary positron spectrum using a diffusive model for Galactic cosmic-ray propagation is described by Moskalenko \& Strong (1998). If the locally observed nucleon spectrum is used, then the expected positron fraction is in good agreement with the measurements up to $10 \mathrm{GeV}$, beyond which the observed flux is higher than the calculated one (Moskalenko \& Strong 1998). From EGRET observations, on the other hand, diffuse Galactic $\gamma$-ray data (Hunter et al. 1997) indicate that the $\gamma$-ray spectrum is harder than expected for the locally measured nucleon spectrum. In order to fit the EGRET data, the spectral index of the nucleon spectrum is required to be 2.41-2.55, and this result cannot be explained in conventional models of cosmic-ray propagation and from cosmicray anisotropy arguments (Mori 1997). Furthermore, detailed studies indicate that antiproton and positron data provide rather substantial evidence against the idea of explaining the $>1 \mathrm{GeV} \gamma$-ray excess with a hard nucleon spectrum (Moskalenko et al. 1998; Strong et al. 2000). Therefore, there may be other sources for the cosmic-ray positrons. 
Many models have been proposed to explain the excess of the cosmic-ray positron fraction with respect to the prediction from the secondary sources. It has been proposed that cosmic-ray positrons with high-energies are produced by annihilating Galactic-halo dark matter weakly-interacting massive particles (see, for example, Kamionkowski \& Turner 1991; Jungman et al. 1996; Baltz \& Edsjö 1999; Moskalenko \& Strong 1999), or by the interaction of hadronic cosmic rays with giant molecular gas clouds (Dogiel \& Sharov 1990), or by pair creation near discrete sources such as Galactic pulsars etc. (for example, Harding \& Ramaty 1987; Chi et al. 1996). However, Coutu et al. (1999) compared these model results with the observed data and concluded that none of these models can be ruled out yet because of the uncertainties in the models and in the observed data (Coutu et al. 1999). Here we will focus on the origin of cosmic ray positrons from mature pulsars.

It has been proposed that pulsars are possible sources of the cosmic positrons by many authors (e.g. Harding \& Ramaty 1987; Boulares 1989; Aharonian et al. 1995; Chi et al. 1996). Harding \& Ramaty (1987) estimated the young pulsar contribution to galactic cosmic ray positrons. Assuming that all Galactic pulsars emit $\gamma$-rays (like the Crab and Vela pulsars) up to a time $\sim 10^{4}$ yr after their birth, and that the positrons are produced with the $\gamma$-rays in cascades near the polar cap of the pulsars, they estimated the total galactic positron production from the Galactic pulsars. Then, using the "leaky box" model of cosmic-ray propagation, they found that the pulsar positron component has a flatter spectrum than that expected from secondary cosmic ray production, and the positron fraction should be approach 0.5 at high energies. Boulares (1989) examined the observed data of the cosmic electron spectrum and positron fraction spectrum above $1 \mathrm{GeV}$, and suggested that the Crab-like supernova remnants and their pulsars probably produce a flatter spectrum, which is negligible at low energies but dominates the total spectrum at high energies. Aharonian et al. (1995) proposed a two-component model of cosmic ray propagation to explain the observed excess of the cosmic positron fraction. In their model, there are two components of the primary electrons: (i) the contribution from one or a few nearby local sources (L-component), and (ii) the contribution from sources at large distances (typically beyond $1 \mathrm{kpc}$ ), which may still be treated in the frame of the traditional assumption of a uniform and continuous source distribution in the Galaxy (G-component). They estimated the positron fraction, which increases with energy beyond about $4 \mathrm{GeV}$ and approaches $\sim 0.5$ at $1000 \mathrm{GeV}$. They argued that the nearby $\gamma$-ray pulsar Geminga is a probable source responsible for the observed very high energy electrons. However, it is generally argued that young pulsars should be surrounded by their nebulae, so it seems that the positrons do not come from the pulsar directly because these positrons should lose their energies through synchrotron radiation and through inverse Compton scattering before they escape from the nebula. So another pos- sibility is that the cosmic ray positrons may come from mature $\gamma$-ray pulsars with ages of $\geq 10^{5}$ yrs, as pointed out by Chi et al. (1996). For these pulsars, it is likely that they are not surrounded by nebulae. In fact, Becker et al. (1999) have analyzed Geminga, PSR B1055-52 and PSR B0656+14 using X-ray data from ROSAT and ASCA observations, and found that there are no nebulae around these three pulsars, which are mature pulsars with ages of $\geq 10^{5}$ yrs. Chi et al. (1996) proposed that old pulsars with their ages of $>10^{5} \mathrm{yr}$ in the Galaxy are a possible source of cosmic-ray electrons and positrons. In their model, the $\mathrm{e}^{ \pm}$pairs escaping from the light cylinder have two components. One is the secondary $\mathrm{e}^{ \pm}$pairs which are the secondary products of primary $\mathrm{e}^{ \pm}$pairs accelerated in the polar gap. Another is the $\mathrm{e}^{ \pm}$pair component in a synchrotron photon cascade of return high-energy $\mathrm{e}^{ \pm}$pairs from the outer gap. These $\mathrm{e}^{ \pm}$pairs escaping from the light cylinder for a single pulsar can be accelerated monoenergetically to relativistic energies in the wind driven by low-frequency electromagnetic waves. Based on the pair production rate of the individual pulsar and the Galactic pulsar population, Chi et al. (1996) estimated the electron-positron flux from the Galactic pulsars and showed that the predicted flux can account for the measured positron fraction. Here, we revisit the mature $\gamma$-ray pulsar contribution to galactic cosmic ray positrons. We assume that the $\mathrm{e}^{ \pm}$pairs escaping from the light cylinder are accelerated in the pulsar wind driven by low-frequency electromagnetic waves, as pointed out by Chi et al. (1996). However, there are two main differences of our treatment to that given by Chi et al. (1996). The first is the estimate of the $\mathrm{e}^{ \pm}$pair production in the pulsar magnetosphere; here, we will use the outer gap model proposed by Zhang \& Cheng (1997). This model has been applied successfully to explain the $\mathrm{X}$-ray and $\gamma$-ray emission from the rotation-powered pulsars (Zhang \& Cheng 1998; Cheng et al. 1998; Cheng \& Zhang 1999) as well as the statistics of Galactic $\gamma$-ray pulsars (Cheng \& Zhang 1998). In this model, a small fraction of $\mathrm{e}^{ \pm}$pairs produced in the cascade of the return $\mathrm{e}^{ \pm}$pairs from the outer gap can escape from the light cylinder. The second is that our statistical analysis of the Galactic pulsar population is different from that of Chi et al. Chi et al. (1996) used the observed sample of radio pulsars with ages greater than $10^{5} \mathrm{yrs}$, while we will use the Monte Carlo simulations of Galactic pulsars which follow our previous works (Cheng \& Zhang 1998; Zhang \& Cheng 1998). This difference is very important because we have demonstrated that the distribution of the radio-selected pulsars and the intrinsic distribution of $\gamma$-ray pulsars, which contributed to most positrons, are different (Zhang et al. 2000). Briefly, we will use a new outer gap model of gamma-ray pulsars and a statistical method different from that used by Chi et al. (1996), all of which will give a different result to that given by Chi et al. (1996). In Sect. 2, we describe our model and estimate the positron production rate from Galactic $\gamma$-ray pulsars. In Sect. 3, we calculate the cosmic-ray positron 
fraction and compare it with the observed data. Finally, we discuss our findings in Sect. 4.

\section{The model}

\subsection{Pair production of a single $\gamma$-ray pulsar}

Zhang \& Cheng (1997) have proposed a self-consistent outer gap model for $\gamma$-ray emission from pulsars. In this model, half of the primary $\mathrm{e}^{ \pm}$pairs in the outer gap will move toward the star and lose their energy via the curvature radiation. Although most of the energy of the primary particles will be lost on the way to the star via curvature radiation, about $10.6 P^{1 / 3}$ ergs per particle will be deposited on the stellar surface and produce X-rays with a typical energy of $E_{\mathrm{X}}^{\mathrm{h}} \approx 1.2 P^{-1 / 6} B_{12}^{1 / 4} \mathrm{keV}$ from a hot polar cap, where $P$ is the pulsar period in seconds and $B_{12}$ is the surface field strength in units of $10^{12} \mathrm{G}$. These $\mathrm{X}$-rays from a hot polar cap will be reflected back to the stellar surface due to the cyclotron resonance scattering in a dense $\mathrm{e}^{ \pm}$pair layer. Eventually, soft thermal X-rays with a typical energy of $E_{\mathrm{X}}^{\mathrm{s}} \approx 0.1 f^{1 / 4} P^{-1 / 4} E_{\mathrm{X}}^{\mathrm{h}}$ will be emitted from the stellar surface. These soft photons control the size of the outer gap by photon-photon pair production in the outer gap. The fraction size of the outer gap is given by

$f=5.5 P^{26 / 21} B_{12}^{-4 / 7}$.

From Eq. (1), if the fraction size of a pulsar is not larger than 1 , then this pulsar is a $\gamma$-ray pulsar, otherwise it is not a $\gamma$-ray pulsar. According to Zhang \& Cheng (1997), the return charged particles from the outer gap will lose their energies through curvature radiation. The Lorentz factor of these charged particles at the distance $r$ near the stellar surface is given by

$\gamma_{\mathrm{e}}(r) \approx 1.510^{7} P^{1 / 3}\left[\ln \left(\frac{r_{\text {in }}}{r}\right)\right]^{-1 / 3}$,

where $r_{\text {in }}$ is the inner boundary of the outer gap. The corresponding energy can be written as

$E_{\mathrm{e}}(r) \approx 12.3 P^{1 / 3}\left[\ln \left(\frac{r_{\text {in }}}{r}\right)\right]^{-1 / 3}$ ergs.

These primaries will emit curvature photons with a typical energy of

$E_{\text {cur }}=\frac{3}{2}\left(\frac{E_{\mathrm{e}}(r)}{m c^{2}}\right)^{3} \frac{c}{s} \hbar$

where $s$ is the radius of curvature, $m$ is the electron mass, $c$ is the light speed and $\hbar$ is the Planck constant. These curvature photons will be converted into secondary $\mathrm{e}^{ \pm}$pairs if their energies satisfy

$E_{\text {cur }} \geq \frac{2 m c^{2}}{15} \frac{B_{q}}{B} \equiv E_{\text {crit }}=3 B_{12}^{-1} \mathrm{MeV}$,

where $B_{q}=4.410^{13} \mathrm{G}$ and $B_{12}$ is the magnetic field in units of $10^{12} \mathrm{G}$. The secondary $\mathrm{e}^{ \pm}$pairs will lose their energy through synchrotron radiation with a characteristic energy

$$
E_{\mathrm{syn}}=\frac{3}{2}\left(\frac{E_{\mathrm{cur}}}{2 m c^{2}}\right)^{2} \frac{e \hbar B(r)}{m c} .
$$

Usually, $E_{\mathrm{syn}}>E_{\text {crit }}$, so a cascade will occur. On average, each incoming primary electron/positron can produce

$N_{\mathrm{e}^{ \pm}} \approx \frac{E_{\mathrm{e}}(r)}{E_{\text {crit }}}$

secondary $\mathrm{e}^{ \pm}$pairs. The total pair production rate can be approximated as

$\dot{N}_{\mathrm{e}^{ \pm}}=f \dot{N} N_{\mathrm{e}^{ \pm}}$

where $\dot{N} \approx 2.710^{30} P^{-2} B_{12}$ is the Goldreich-Julian number per unit time. However, only part of these $\mathrm{e}^{ \pm}$pairs can move toward the light cylinder along the open magnetic field lines, and finally escape from the light cylinder. We introduce a parameter $\zeta$ to describe the number of the $\mathrm{e}^{ \pm}$pairs escaping from the light cylinder. We estimate $\zeta$ as follows. The cascade of $\mathrm{e}^{ \pm}$pairs start begins at $r_{\mathrm{s}} / R \approx 0.6 B_{12}^{1 / 3}\left(E_{\mathrm{cur}} / m c^{2}\right)^{1 / 3}$, which is determined by $\left(E_{\text {cur }} / 2 m c^{2}\right)\left(\hbar e B\left(r_{\mathrm{s}}\right) / m c\right)>1 / 15$, where $R$ is the stellar radius (e.g. Cheng \& Zhang 1999). The hard X-rays from the polar cap will scatter with these $\mathrm{e}^{ \pm}$pairs and push them away from the star via resonant scattering (Halpern \& Ruderman 1993). In the closed field lines, these pairs will form a $\mathrm{e}^{ \pm}$blanket near the stellar surface (Wang et al. 1998). However, in the open field lines, the hard X-rays can push the cascade $\mathrm{e}^{ \pm}$pairs along the open field lines to eventually leave the light cylinder. Therefore, the parameter $\zeta$ can be estimated as

$\zeta \sim 2 \frac{2 \pi\left(1-\cos \theta\left(r_{\mathrm{s}}\right)\right)}{4 \pi} \approx \frac{\theta^{2}\left(r_{\mathrm{s}}\right)}{2} \approx \frac{1}{2} \frac{r_{\mathrm{s}}}{R_{\mathrm{L}}}$,

where $R_{\mathrm{L}}$ is the light cylinder radius of the pulsar. According to Cheng \& Zhang (1999), $r_{\mathrm{s}} / R_{\mathrm{L}} \sim$ $7.510^{-3} f^{1 / 2} B_{12}^{7 / 12} P^{-19 / 12}\left(r_{\text {in }} / R_{\mathrm{L}}\right)^{-0.54}$, where $r_{\text {in }}$ is the radius of inner boundary of the outer gap, which depends on the magnetic inclination angle $\alpha,\left(r_{\text {in }} / R_{\mathrm{L}}\right) \approx$ $4(\pi / 2-\alpha) / 9$ (Romani 1996). So we have

$\zeta \sim 3.810^{-4} f^{1 / 2} B_{12}^{7 / 12} P^{-19 / 12}\left(\frac{4(\pi / 2-\alpha)}{9}\right)^{-0.54}$.

For a typical pulsar (e.g. Geminga, $r_{\mathrm{s}} \sim 6 R$ ), $\zeta \sim 10^{-2}$. Therefore, the number of $\mathrm{e}^{ \pm}$pairs just outside the light cylinder is given by

$\dot{N}_{\mathrm{e}^{ \pm}}=\zeta \dot{N}_{\mathrm{e}^{ \pm}}=\zeta f \dot{N} N_{\mathrm{e}^{ \pm}}$.

It should be pointed out that Chi et al. (1996) made an unreasonable assumption: all $\mathrm{e}^{ \pm}$pairs will escape from the light cylinder (i.e. $\zeta=1$ ). Outside the light cylinder, the $\mathrm{e}^{ \pm}$pairs move out with the pulsar wind. The plasma frequency of these $\mathrm{e}^{ \pm}$pairs at the distance $d$ to the pulsar is $f_{\mathrm{p}}=\left(4 \pi n_{ \pm} \mathrm{e}^{2} / m\right) / 2 \pi$ with $n_{ \pm}=\dot{N}_{\mathrm{e}^{ \pm}}^{\prime} / 4 \pi d^{2} c$. It can be shown that $f_{p} \gg P^{-1}$, so electromagnetic waves with 
low frequencies will push $\mathrm{e}^{ \pm}$plasma until $f_{\mathrm{p}}=P^{-1}$, or the equipartition between the electromagnetic waves and the kinetic energy of the charged particles is reached (Chi et al. 1996), i.e.

$n_{ \pm}(d) E_{\mathrm{e}}=\frac{B^{2}(d)}{4 \pi c}$

with

$B(d)=B_{\mathrm{s}}\left(\frac{R}{R_{\mathrm{L}}}\right)^{3}\left(\frac{R_{\mathrm{L}}}{d}\right)$,

where $B_{\mathrm{S}}$ is the surface magnetic field and $R_{\mathrm{L}}$ is the radius of the light cylinder. From Eq. (12), the equipartition energy of the accelerated charged particles can be determined by

$E_{\text {equi }} \approx 6.110^{8} f^{-1} \zeta_{-2}^{-1} P^{-7 / 3} \mathrm{eV}$,

where $\zeta_{-2}=\zeta / 0.01$. As pointed out by Chi et al. (1996), the low-frequency electromagnetic waves quickly transfer their energies to $\mathrm{e}^{ \pm}$pairs via magnetic field reconnection (Coroniti 1990), and the equipartition can rapidly be achieved before the magnetic connection is completed in the pulsar wind (for Crab, the expected escape distance is about one order of magnitude larger than the equipartition distance, see Coroniti 1990). Therefore, adiabatic energy loss should be taken into account. Chi et al. (1996) assumed that particle energy ejected into the interstellar medium is one order of magnitude lower than the equipartition energy give by Eq. (14). Here we will use this assumption. In other words, the ejected electron/positron energy is about $E_{\mathrm{e}} \sim 0.1 E_{\text {equi }}$. The corresponding number of electrons/positrons per unit time and per unit energy is given by

$Q_{\mathrm{e}^{ \pm}}=\frac{\dot{N}_{\mathrm{e}^{ \pm}}}{E_{\mathrm{e}}} \approx 9.710^{35} \zeta_{-2}^{2} f^{2} B_{12}^{2} P^{2 / 3} \quad \mathrm{GeV}^{-1} \mathrm{~s}^{-1}$

It should be noted that the $\mathrm{e}^{ \pm}$pair production rate per unit energy for a given pulsar can be estimated using Eq. (15).

\section{2. $e^{+}$production rate of Galactic mature $\gamma$-ray pulsars}

In the previous section, we gave the positron production rate from a single $\gamma$-ray pulsar. In order to estimate the positron production rate of Galactic mature $\gamma$-ray pulsars, we use Monte Carlo code of galactic $\gamma$-ray pulsar population (Cheng \& Zhang 1998; Zhang \& Cheng 1998) to generate a mature $\gamma$-ray pulsar sample. The pulsar ages are from $10^{5}$ yrs to $210^{7}$ yrs in this sample. In our simulations, the following assumptions for generating the Galactic pulsar population are used. (i) The pulsars are born at a rate $\left(\dot{N}_{\mathrm{NS}} \sim(1-2)\right.$ per century) with spin periods of $P_{0}=$ $30 \mathrm{~ms}$. (ii) The initial position for each pulsar is estimated from the distributions $\rho_{z}(z)=\left(1 / z_{\exp }\right) \exp \left(-|z| / z_{\exp }\right)$ and $\rho_{R}(R)=\left(a_{R} / R_{\text {exp }}^{2}\right) R \exp \left(-R / R_{\exp }\right)$, where $z$ is the distance from the Galactic plane, $R$ is the distance from the
Galactic center, $z_{\exp }=75 \mathrm{pc}, a_{R}=\left[1-\mathrm{e}^{-R_{\max } / R_{\exp }}(1+\right.$ $\left.\left.R_{\max } / R_{\exp }\right)\right]^{-1}, R_{\exp }=4.5 \mathrm{kpc}$ and $R_{\max }=20 \mathrm{kpc}$ (Paczynski 1990; Sturner \& Dermer 1996). (iii) The initial magnetic fields are distributed as a Gaussian in $\log B$ with mean $\log B_{0}=12.4$ and dispersion $\sigma_{B}=0.3$. We ignore any field decay for these rotation-powered pulsars. (iv) The initial velocity of each pulsar is the vector sum of the circular rotation velocity at the birth location and a random velocity from the supernova explosion (Paczynski 1990; Cheng \& Zhang 1998); the circular velocity is determined by Galactic gravitational potential and the random velocity is distributed as a Maxwellian distribution with a dispersion of the three dimensional velocity $\sigma_{\mathrm{V}}=\sqrt{3} 100 \mathrm{~km} \mathrm{~s}^{-1}$ (Lorimer et al. 1997). (v) A random distribution of magnetic inclination angles is assumed. Using these initial properties of a pulsar at birth, the pulsar period at time $t$ can be estimated by

$P(t)=\left[P_{0}^{2}+\left(\frac{16 \pi^{2} R_{\mathrm{NS}}^{6} B^{2}}{3 I c^{3}}\right) t\right]^{1 / 2}$,

where $R_{\mathrm{NS}}$ is the neutron star radius and $I$ is the neutron star moment of inertia. The period derivative $(\dot{P})$ can be determined by

$P \dot{P}=\left(8 \pi^{2} R_{\mathrm{NS}}^{6} / 3 I c^{3}\right) B^{2}$.

Furthermore, the pulsar position at time $t$ is determined following its motion in the Galactic gravitational potential. Using the Monte Carlo simulation, we follow each pulsar with its initial period, position, magnetic field and velocity. A pulsar is a $\gamma$-ray pulsar if its fraction size (Eq. (1)) is not larger than 1 . We record the energy and pair production rate of each $\gamma$-ray pulsar with an age of greater than $10^{5}$ yrs. We then obtain a distribution of the positron production rate with energy, which is

$Q_{\mathrm{e}^{+}}^{\mathrm{psr}}(E)=\sum_{i=1}^{N} Q_{\mathrm{e}^{+}}^{\mathrm{psr}, i}\left(E_{i}\right)$

where $Q_{\mathrm{e}^{+}}^{\mathrm{psr}, i}\left(E_{i}\right)$ is the positron production rate of the $i$ th $\gamma$-ray pulsar with energy $E_{i}$. Harding \& Ramaty (1987) have estimated the total galactic positron production rate from the Galactic pulsars, which is $Q^{\mathrm{psr}}(E)=$ $8.610^{39} \dot{N}_{30} f_{+} B_{12}^{-0.7}\left(t_{\max } / 10^{4} \mathrm{yr}\right)^{0.15} E^{-2.2} \mathrm{GeV}^{-1} \mathrm{~s}^{-1} \mathrm{~g}^{-1}$, where $\dot{N}_{30}$ is the birth rate of the neutron stars in units of one per $30 \mathrm{yrs}$, and $f_{+}$is the ratio of positrons to $\gamma$-rays produced by a pulsar. Using the parameters given by Hardiang \& Ramaty (1987), we have $q^{\mathrm{psr}}(E) \approx 1.810^{39} E^{-2.2} \mathrm{GeV}^{-1} \mathrm{~s}^{-1}$. As a comparison, we also show the result of Harding \& Ramaty in Fig. 1.

In Fig. 1, we show the positron production rate distribution of the galactic mature $\gamma$ pulsars with a birth rate of $\dot{N}_{100}=1$ (i.e. the neutron stars are born at the rate of one per one hundred). From Fig. 1, the positron production rate $\left(Q_{\mathrm{e}^{+}}^{\mathrm{psr}}\right)$ peaks at about $1 \mathrm{GeV}$. It drops rapidly below this energy. Above about $1 \mathrm{GeV}$, it decrease with an approximate power-law and has a cut-off at about $100 \mathrm{GeV}$. It can be seen that $Q_{\mathrm{e}^{+}}^{\mathrm{psr}} \propto E^{-1.6} \exp (-E / 80)$ 


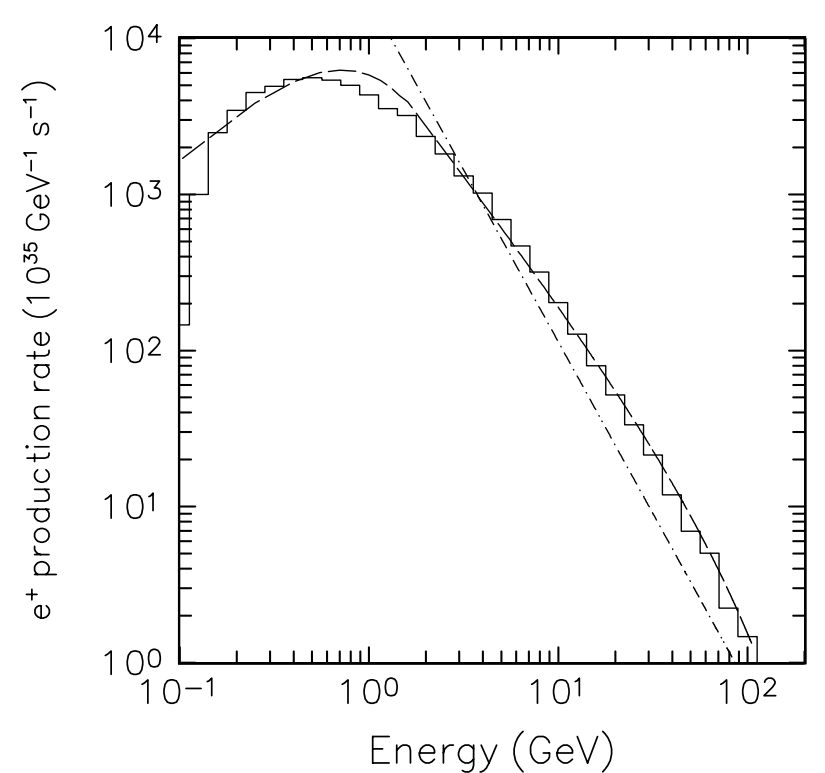

Fig. 1. The positron production rate from $\gamma$-ray pulsar winds in the Galaxy. Histogram and the long-dashed curve represent the positron production rate from mature $\gamma$-ray pulsar winds, where neutron star birth rate is assumed to be one per $100 \mathrm{yrs}$. Dotted-dashed curve represents the result given by Harding \& Ramaty (1987)

for $E \geq 1.5 \mathrm{GeV}$, where $E$ is positron energy. Therefore, the positron production rate for $E \geq 1.5 \mathrm{GeV}$ can be approximated as

$q_{\mathrm{e}^{+}}^{\mathrm{psr}}(E) \approx 4.310^{-5} \dot{N}_{100} E^{-1.6} \exp \left(-\frac{E}{80}\right) \frac{\mathrm{GeV}^{-1}}{\mathrm{~g} \mathrm{~s}}$.

In Eq. (19), we have taken the total mass of our Galaxy to be $M_{\mathrm{g}} \approx 10^{10} M_{\odot}$, where $M_{\odot}$ is the solar mass.

From Eq. (19), the positron production rate from the mature pulsars in the Galaxy depends on the birth rate of the neutron star, $\dot{N}_{100}$. It is generally believed that the birth rate of neutron stars is from one per 100 yrs to one per 30 yrs. Therefore, the uncertainty of the positron production rate is about a factor of 3 in our model. We would like to point out that our model predicts the spacial distribution of the mature pulsars in the Galaxy. These mature pulsars are concentrated on the Galactic disk, and their radial distribution can be approximated as $f(R) \propto$ $(R / 8) \exp (-1.8(R-8) / 8)$.

\section{Cosmic-ray positron fraction}

Once the positron production rate is known, the positron propagation in our Galaxy can be described in "leaky box" or diffusion models (e.g. Protheroe 1982; Moskalenko \& Strong 1998). In order to compare our predictions with observed data, we have to consider the expected spectra of cosmic-ray electrons and positrons with pure secondary origin, i.e. primary electron, secondary electron and secondary positron fluxes. In principle, the diffusion model is more realistic than the leaky box model to describe cosmic-ray propagation in the Galaxy. In the dif- fusion model, the spatial properties, such as structure of the Galaxy, the spatial distributions of source function and interstellar radiation and magnetic fields are taken into account. These spatial properties are ignored in the leaky box model; instead, the leaky box model assumes a homogeneous spatial distribution (see, for example, Berezinsky et al. 1990). Under some assumptions that positron function is dependent only on the radial cylinder coordinate and that the interstellar radiation and magnetic field is spatially uniform, Baltz \& Edsjö (1999) gave an analytic solution of the diffusion model for cosmic-ray positron propagation. Moskalenko \& Strong (1999) calculated propagation of cosmic-ray electrons and positrons based on their numerical method and corresponding computer code for the calculation of Galactic cosmic-ray propagation in 3D (Strong \& Moskalenko 1998). They pointed out that a reliable background evaluation requires new accurate positron measurements and further developments in modeling production and propagation of cosmic ray species in the Galaxy. Both models predict the same behavior of the positron fractions, i.e. a smooth, monotonic decrease without a spectral feature (Coutu et al. 1999). Because of the limited statistics and absence of accurate positron data at high energies we will use the leaky-box model, which allows us to qualitatively estimate propagation of primary positrons.

\subsection{Cosmic-ray electron and positron background}

To compare our predictions with the observed data of the cosmic-ray positron fraction, we need to know the background spectra of cosmic-ray electrons and positrons respectively. It is generally believed that primary cosmic-ray electrons dominate in the cosmic-ray electron background and the positron background is formed by secondary products of nuclear interactions of cosmic ray with the interstellar medium. In the following, we use the recent calculation (08-005 without reacceleration) by Moskalenko \& Strong (1998) as the background spectra of cosmic ray electrons and positrons. Baltz \& Edsjö (1998) have parametrized these spectra as follows:

$$
\begin{aligned}
& J_{\mathrm{e}^{-}, \operatorname{prim}}(E)= \\
& \frac{0.16 \epsilon^{-1.1}}{1+11 \epsilon^{0.9}+3.2 \epsilon^{2.15}} \mathrm{GeV}^{-1} \mathrm{~cm}^{-2} \mathrm{~s}^{-1} \mathrm{sr}^{-1}, \\
& J_{\mathrm{e}^{-}, \mathrm{sec}}(E)= \\
& \frac{0.7 \epsilon^{0.7}}{1+470 \epsilon^{1.7}+240 \epsilon^{2.9}+580 \epsilon^{4.2}} \mathrm{GeV}^{-1} \mathrm{~cm}^{-2} \mathrm{~s}^{-1} \mathrm{sr}^{-1},
\end{aligned}
$$

$$
\begin{aligned}
& J_{\mathrm{e}^{+}, \mathrm{sec}}(E)= \\
& \frac{4.5 \epsilon^{0.7}}{1+650 \epsilon^{2.3}+1500 \epsilon^{4.2}} \mathrm{GeV}^{-1} \mathrm{~cm}^{-2} \mathrm{~s}^{-1} \mathrm{sr}^{-1},
\end{aligned}
$$

where $\epsilon=E /(1 \mathrm{GeV})$. It should be noted that we have corrected the formula given by Baltz \& Edsjö (1998) for secondary electrons. 


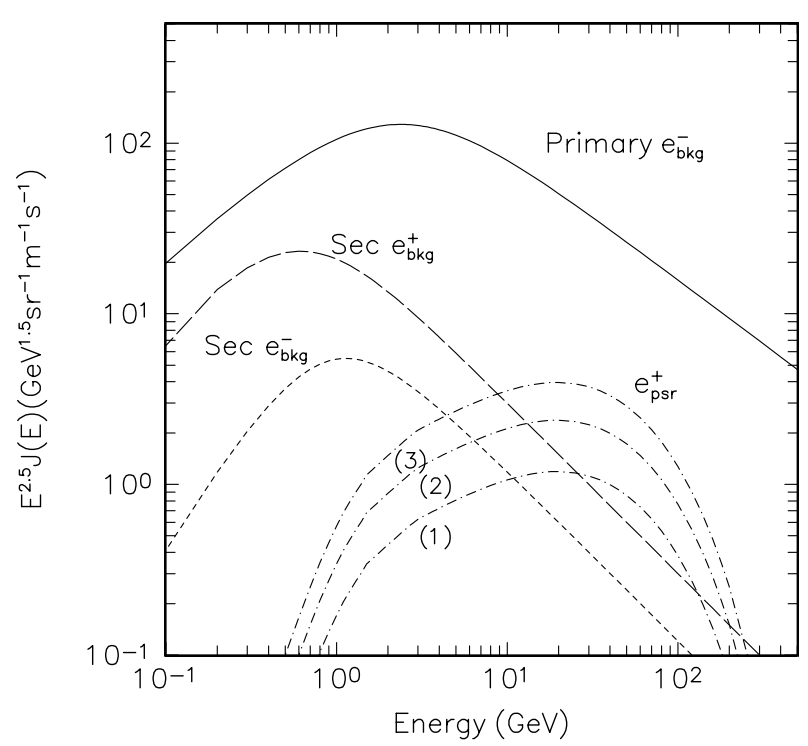

Fig. 2. The spectra of cosmic-ray electrons and positrons. The background of cosmic-ray electrons and positrons are approximated by those given by Moskalenko \& Strong (1998): primary $\mathrm{e}_{\mathrm{bkg}}^{-}$(solid curve), secondary $\mathrm{e}_{\mathrm{bkg}}^{-}$(short-dashed curve) and secondary $\mathrm{e}_{\mathrm{bkg}}^{+}$(long-dashed curve). The positron spectra from the mature $\gamma$-ray pulsars for various birth rate of the neutron stars in the Galaxy are labeled as $(1)\left(\dot{N}_{100}=1\right),(2)\left(\dot{N}_{100}=2\right)$ and $(3)\left(\dot{N}_{100}=10 / 3\right)$

\subsection{Positron fraction}

We now estimate the cosmic-ray positron fraction. If cosmic-rays are of pure secondary origin, the positron fraction is approximated as $J_{\mathrm{e}^{+}, \mathrm{sec}}(E) /\left(J_{\mathrm{e}^{-}, \operatorname{prim}}(E)+\right.$ $\left.J_{\mathrm{e}^{-}, \text {sec }}(E)+J_{\mathrm{e}^{+}, \text {sec }}(E)\right)$. In order to include the possible contribution from the mature $\gamma$-ray pulsars, we estimate the positron spectrum from the mature $\gamma$-ray pulsars in the leaky box model. In the leaky box model, the spectrum of cosmic-ray electrons/positrons is given by

$$
\begin{aligned}
J_{\mathrm{e}^{ \pm}}^{\mathrm{psr}}(E)= & \frac{1}{4 \pi}\left(\frac{\mathrm{d} E}{\mathrm{~d} x}\right)^{-1} \int_{E}^{\infty} \mathrm{d} E^{\prime} q_{\mathrm{e}^{+}}^{\mathrm{psr}}(E) \\
& \times \exp \left[-\int_{E}^{E^{\prime}} \frac{\mathrm{d} E^{\prime \prime}}{\lambda_{\mathrm{e}}\left(E^{\prime \prime}\right)(\mathrm{d} E / \mathrm{d} x)}\right],
\end{aligned}
$$

where $\mathrm{d} E / \mathrm{d} x \approx 510^{-3} E^{2}$ is electron (or positron) energy loss in units of $\mathrm{GeV} \mathrm{cm}^{2} \mathrm{~g}^{-1}$, including synchrotron radiation and inverse Compton scattering, $\lambda_{\mathrm{e}}(E)$ is energydependent escape length in units of $\mathrm{g} \mathrm{cm}^{-2}$, which is $\lambda_{\mathrm{e}}(E)=7.0$ for $R \leq 4 \mathrm{GV}$ and $\lambda_{\mathrm{e}}(E)=7(R / 4)^{-0.4}$ for $R>4 \mathrm{GV}$, where $R$ is particle rigidity. $q_{\mathrm{e}}(E)$ is the production rate of electrons (or positrons) in units of $\mathrm{GeV}^{-1} \mathrm{~g}^{-1} \mathrm{~s}^{-1}$. In Fig. 2, we show the background spectra of cosmic-ray electrons and positrons and the positron spectra from the mature $\gamma$-ray pulsars for different birth rate of the neutron stars in the Galaxy.

Further, we estimate the cosmic-ray positron fraction which includes the contribution of the Galactic mature $\gamma$ ray pulsars using $\left(J_{\mathrm{e}^{+}, \mathrm{sec}}(E)+J_{\mathrm{e}^{+}, \mathrm{psr}}(E)\right) /\left(J_{\mathrm{e}^{-}, \operatorname{prim}}(E)+\right.$ $\left.J_{\mathrm{e}^{-}, \mathrm{sec}}(E)+J_{\mathrm{e}^{+}, \mathrm{sec}}(E)+J_{\mathrm{e}^{+}, \mathrm{psr}}(E)+J_{\mathrm{e}^{-}, \mathrm{psr}}(E)\right)$. The comparison of our results with the observed data is shown in
Fig. 3. In the leaky box model, we show the predicted results for different pulsar birth rates, which are consistent with the observed data. Generally, the mature $\gamma$-ray pulsar contribution to cosmic ray positrons becomes important above $\sim 4 \mathrm{GeV}$; the predicted $\mathrm{e}^{+} /\left(\mathrm{e}^{-}+\mathrm{e}^{+}\right)$ratio in the high energy range peaks at about $60 \mathrm{GeV}$. Although the above results are obtained within the leaky box model, they indicate that Galactic $\gamma$-ray pulsar contribution has an important role in the cosmic-ray positron fraction at high energy range. It can be seen that the positron fraction indicates a smooth, monotonic decrease without spectral features (Coutu et al. 1999). Therefore, the observed excess of the cosmic-ray positron fraction at high energy range cannot be explained in both the leaky box model and diffusion model if the positrons are assumed to be of purely secondary origin and produced by the interaction of cosmic-ray nuclei with interstellar matter. The positrons from Galactic $\gamma$-ray pulsars discussed here may provide a possible explanation.

It should be pointed out that solar modulation has an important role in the cosmic-ray electron and positron fluxes with energies less than $10 \mathrm{GeV}$. If the charge-sign dependence is absent, then the cosmic-ray positron fraction is unaffected by solar modulation effects. However, the charge-sign dependent solar modulation will influence the change in the positron fraction, especially in the low energy range (i.e. $\leq 10 \mathrm{GeV}$ ), as pointed out by Clem et al. (1996). Here, we did not consider the solar modulation effect.

\section{Conclusions and discussion}

We have estimated the positron contribution from the mature $\gamma$-ray pulsars to cosmic ray positrons. Although positrons are monoenergetical for each pulsar, the production rate for all mature $\gamma$-ray pulsars in the Galaxy satisfies a distribution shown in Fig. 1. Particularly, it is proportional to $E^{-1.6} \exp (-E / 80)$ from $\sim 1.5 \mathrm{GeV}$ to $\sim 100 \mathrm{GeV}$ and drops abruptly above $\sim 100 \mathrm{GeV}$. We find that the predicted cosmic ray positron fraction from the mature pulsars for reasonable pulsar birth rates are consistent with the observed data (see Fig. 3). It should be pointed out that we have only considered the positrons from the mature $\gamma$-ray pulsars. Generally, young $\gamma$-ray pulsars can also produce abundant $\mathrm{e}^{ \pm}$pairs in the pulsar magnetosphere. However, it is believed that pulsars are born in the explosion of supernova, so young pulsars are immersed in the supernova remnant, because the speed of pulsar proper motion is normally 1 or 2 orders of magnitude less than that of the initial supernova blast wave. Therefore, the $\mathrm{e}^{ \pm}$pairs accelerated by pulsar winds should lose their energies via synchrotron radiation, which can emit high-energy photons from X-rays to $\gamma$-rays (e.g. see de Jager \& Harding 1992 for Crab). Theoretically, Slavin \& Cox (1992) showed that the supernova expansions in the warm interstellar medium will slow down significantly after $10^{5} \mathrm{yrs}$. Therefore the pulsar with a kick velocity $\sim 500 \mathrm{~km} \mathrm{~s}^{-1}$ can leave the supernova 


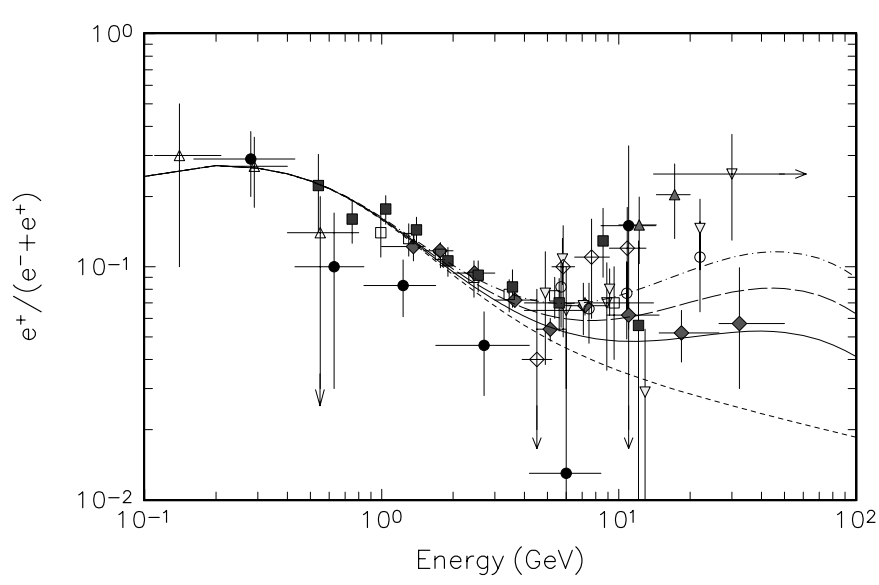

Fig. 3. Comparison of observed $\mathrm{e}^{+} /\left(\mathrm{e}^{-}+\mathrm{e}^{+}\right)$ratio with predicted ones. The short-dashed curve is the ratio of positron background and total electron background given by Moskalenko \& Strong (1998). The solid, long dashed and dotdashed curves are the ratios including the contribution of the mature $\gamma$-ray pulsars with birth rates of one per 100 yrs, one per 50 yrs and one per 30 yrs respectively. The observed data are taken from Fanselow et al. (1969) (solid circles), Daugherty et al. (1975) (open up-triangles), Buffington et al. (1975) (open down-triangles), Müller \& Tang (1987) (solid uptriangles), Golden et al. (1987) (solid down-triangles), Golden et al. (1994) (open diamonds), Golden et al. (1996) (open circles), Barbiellini et al. (1996) (open boxes), Barwick et al. (1997) (solid diamonds), and Boezio et al. (2000) (solid boxes)

remnant. Observationally, Becker et al. (1999) found the mature pulsars such as Geminga, PSR B1055-52 an PSR B0656+14 are not surrounded by nebulae. Therefore, energetic $\mathrm{e}^{ \pm}$pairs from the $\gamma$-ray pulsars may contribute to cosmic ray positrons. In our model, the positron production rate can be estimated for a single mature $\gamma$-ray pulsar using Eq. (15), and the total positron production rate of mature $\gamma$-ray pulsars in the Galaxy can be estimated from our simulations (see Fig. 1), which only depend on the pulsar birth rate $\dot{N}_{100}$ and can be approximated by Eq. (19) for $E \geq 1.5 \mathrm{GeV}$. Furthermore, the cosmic-ray electron and positron backgrounds are approximated by Eqs. (20) to $(22)$; the contribution of positrons from Galactic mature $\gamma$-ray pulsars to cosmic-ray positrons has been estimated in the leaky box model (see Figs. 2 and 3) and may explain the observed excess of positron fraction in reasonable pulsar birth rates. Compared to recent observed data (e.g. Barwick et al. 1997; Coutu et al. 1999; Boezio et al. 2000), a pulsar's birth rate seems most likely to be from $\dot{N}_{100}=1$ to $\dot{N}_{100}=2$.

Our result is different from that given by Chi et al. (1996). The main reasons resulting in the difference have been mentioned in the introduction. In our model, because only a small fraction $(\zeta \sim 0.01)$ of these pairs escape from the light cylinder, the Galactic pulsar contribution to cosmic ray positrons is in the energy range of $\sim 0.1-100 \mathrm{GeV}$. However, Chi et al. (1996) assumed that $\mathrm{e}^{ \pm}$pairs in the pulsar magnetosphere are produced by polar cap and outer gap models respectively; all these pairs escape from the pulsar light cylinder. Therefore, the pulsar contribution to cosmic ray electrons consists of two parts: one is from the outer gap and the other is from polar cap. The former contributes mainly to lower energy $(0.01-\sim 1 \mathrm{GeV})$, and the latter to higher energy $(\sim 1-100 \mathrm{GeV})$. On the other hand, we have used a Monte Carlo simulation to generate a sample of the mature $\gamma$-ray pulsars with outer gaps (i.e. the fraction size of the outer gap must be less than 1 ), but Chi et al. (1996) used just the observed data, which are biased to the selection effects of detectors and assumed that all pulsars are $\gamma$-ray pulsars, which seems unreasonable.

Compare our model result with that given by Harding \& Ramaty (1987). If $q_{\text {cr }}$ represents the positron production rate by cosmic ray interactions per gram of interstellar gas and $q_{\mathrm{cr}} \sim 2.310^{-3} E_{2.7} \mathrm{GeV}^{-1} \mathrm{~s}^{-1} \mathrm{~g}^{-1}$ in the energy range 1 to $100 \mathrm{GeV}$, then Harding \& Ramaty (1987) estimated that the total positron production rate per gram of interstellar medium is $q(E)=q_{\mathrm{cr}}(E)\left(1+k E^{0.5}\right)$. According to our model, the corresponding total positron production rate is $q(E)=q_{\mathrm{cr}}(E)\left(1+1.910^{-2} \dot{N}_{100} E^{1.1} \exp (-E / 80)\right)$. Obviously, the energy dependence of the positron production rate in our model is different from that in the Harding \& Ramaty model, resulting in different predictions. In fact, the Harding \& Ramaty model predicts that the positron fraction should increase with energy beyond $10 \mathrm{GeV}$ and have an asymptotic value of 0.5. Moreover, Aharonian \& Atoyan (1991) argued that positrons can be produced by the interaction of very high energy $\gamma$-rays with optical and /or UV radiation in the vicinity of discrete sources. Aharonian et al. (1995) further proposed that one or a few nearby local sources such as Geminga is a probable source responsible for the observed $\mathrm{e}^{-} /\left(\mathrm{e}^{-}+\mathrm{e}^{+}\right)$. These models predict that the positron fraction should increase with energy beyond $10 \mathrm{GeV}$ and have an asymptotic value of 0.5 , which is different from our prediction.

Acknowledgements. We thank the anonymous referee for his/her constructive comments. This work is partially supported by the Outstanding Researcher Awards of the University of Hong Kong, a Croucher Foundation Senior Fellowship and the National Nature Scientific Foundation of China

\section{References}

Aharonian, F. A., \& Atoyan, A. M. 1991, J. Phys. G, 17, 1769 Aharonian, F. A., Atoyan, A. M., \& Völk, H. J. 1995, A\&A, 294, L41

Baltz, E. A., \& Edsjö, J. 1999, Phys. Rev. D, 59, 023511

Barbiellini, G., Bellotti, R., Bocciolini, M., et al. 1996, A\&A, 309, L15

Barwick, S. W., Beatty, J. J., Bhattacharyya, A., et al. 1997, ApJL, 482, L191

Becker, W., Kawai, N., Brinkmann, W., \& Maignani, R. 1999, A\&A, 352, 532

Berezinsky, V. S., et al. 1990, Astrophysics of Cosmic Rays (North Holland)

Boezio, M. B., Carlson, P., Francke, T., et al. 2000, ApJ, 532, 653

Boulares, A. 1989, ApJ, 342, 807 
Buffington, A., Orth, C. D., \& Smoot, G. F. 1975, ApJ, 199, 669

Cheng, K. S., \& Zhang, L. 1999, ApJ, 515, 337

Cheng, K. S., \& Zhang, L. 1998, ApJ, 498, 327

Chi, X., Cheng, K. S., \& Young, E. C. M. 1996, ApJL, 459, L83

Coutu, S., Barwick, S. W., Beatty, J. J., et al. 1999, Astroparticle Physics, 11, 429

Coroniti, F. V. 1990, ApJ, 538

Daugherty, J. K., Hartman, R. C., \& Schmidt, P. J. 1975, ApJ, 198,493

de Jager, O. C., \& Harding, A. K. 1992, ApJ, 396, 161

Dogiel, V. A., \& Sharov, G. S. 1990, A\&A, 229, 250

Fanselow, J. L., Hartman, R. C., Hildebrand, R. H., \& Meyer, P. 1969, ApJ, 158, 771

Golden, R. L., Mauger, B. G., Horan, S., et al. 1987, A\&A, 188,145

Golden, R. L., Grimani, C., Kimbell, B. L., et al. 1994, ApJ, 436, 769

Golden, R. L., Stochaj, S. J., Stephens, S. A., et al. 1996, ApJL, 457, L103

Halpern, J. P., \& Ruderman, M. 1993, ApJ, 415, 286

Harding, A. K., \& Ramaty, R. 1987, in 20th Int. Cosmic-Ray Conf. (Moscow: Nauka), 2, 92

Hunter, S. D., Bertsch, D. L., Catelli, J. R., et al. 1997, ApJ, 481,205
Jungman, G., \& Kamionkowski, M. 1996, Phys. Rep., 267, 195

Kamionkowski, M., \& Turner, M. S. 1991, Phys. Rev. D, 43, 1774

Lorimer, D. R., Bailes, M., \& Harrison, P. A. 1997, MNRAS, 289, 592

Moskalenko, I. V., \& Strong, A. W. 1999, Phys. Rev. D, 60, 063003

Moskalenko, I. V., Strong, A. W., \& Reimer, O. 1998, A\&A, $338, \mathrm{~L} 75$

Moskalenko, I. V., \& Strong, A. W. 1998, ApJ, 493, 694

Mori, M. 1997, ApJ, 478, 225

Müller, D., \& Tang, K. K. 1987, ApJ, 312, 183

Paczynski, B. 1990, ApJ, 348, 485

Protheroe, R. J. 1982, ApJ, 254, 391

Slavin, J. D., \& Cox, D. P. 1992, ApJ, 392, 131

Strong, A. W., Moskalenko, I. V., \& Reimer, O. 2000, ApJ, 537,763

Sturner, S. J., \& Dermer, C. D. 1996, ApJ, 461, 872

Wang, F. Y.-H., Ruderman, M., Halpern, J. P., \& Zhu, T. 1998, ApJ, 498, 373

Zhang, L., \& Cheng, K. S. 1997, ApJ, 487, 370

Zhang, L., \& Cheng, K. S. 1998, MNRAS, 301, 841

Zhang, L., Zhang, Y. J., \& Cheng, K. S. 2000, A\&A, 357, 967

Zhu, T., \& Ruderman, M. 1997, ApJ, 478, 701 